\title{
A Comparison of Three Voting Methods for Bagging with the MLEM2 Algorithm
}

\author{
Clinton Cohagan ${ }^{1}$, Jerzy W. Grzymala-Busse ${ }^{2,3}$, and Zdzislaw S. Hippe ${ }^{4}$ \\ 1 National Nuclear Security Administration, Kansas City Plant,* \\ Kansas City, MO 64131, USA, \\ ccohagan@kcp.com \\ ${ }^{2}$ Department of Electrical Engineering and Computer Science, University of Kansas, \\ Lawrence, KS 66045, USA \\ 3 Institute of Computer Science, Polish Academy of Sciences, \\ 01-237 Warsaw, Poland \\ jerzy@ku.edu \\ 4 Department of Expert Systems and Artificial Intelligence, \\ University of Information Technology and Management, \\ 35-225 Rzeszow, Poland \\ zhippe@wsiz.rzeszow.pl
}

\begin{abstract}
This paper presents results of experiments on some data sets using bagging on the MLEM2 rule induction algorithm. Three different methods of ensemble voting, based on support (a non-democratic voting in which ensembles vote with their strengths), strength only (an ensemble with the largest strength decides to which concept a case belongs) and democratic voting (each ensemble has at most one vote) were used. Our conclusions are that though in most cases democratic voting was the best, it is not significantly better than voting based on support. The strength voting was the worst voting method.
\end{abstract}

\section{Introduction}

Ensemble learning methods may improve classifier performance. These methods construct an ensemble (a set) of diverse classifiers (resembling the base classifier) that vote on the concept membership. Two methods are used frequently: bagging [1] and boosting [2]. In bagging each classifier is created using different subsets of the training set. These subsets are results of a bootstrap sampling, constructed by drawing cases from the training set with replacement. Thus, a bootstrapped sample has about $63.2 \%$ unique cases from the training set. In boosting, the distribution of training cases is adaptively changed in such a way that the classifier will focus on cases that are difficult to classify. A weight is associated with each case. Weights of previously misclassified cases are increased. Hence, during boosting, more difficult cases are more frequently included in the sample. In both bagging and boosting, final predictions from all classifiers are aggregated. 
Ensemble learning is conducted in two stages: creation of classifiers and combining classifier predictions. Bagging and boosting are typical methods for creating classifiers, but there are many other techniques of ensemble learning, for example, random forest methods [3] in which for every ensemble attributes are randomly selected, see also [4-10]. Usually classifier predictions may be aggregated by voting, stacking [11] and cascading [12]. In stacking, a learning algorithm is applied to classifier predictions. Cascading combines classifier predictions iteratively, in each iteration the training data set is associated with the predictions from previous iterations.

The performance of bagging and boosting, combined with some aggregating mechanisms for combining predictions, is comparable. Bagging is more accurate than variations of random forests, randomized C4.5 [13] and random subspaces [14]. For data sets with many conflicting cases bagging is much better than boosting [15].

For our experiments we used as a base classifier the MLEM2 (Modified Learning from Examples Module, version 2) module of the LERS (Learning from Examples based on Rough Sets) data mining system. Additionally, we used bagging with three different techniques of combining predictions of ensemble classifiers: support, strength, and ordinary voting in which each rule set contributes at most one vote.

\section{MLEM2}

In our experiments, ensembles were rule sets induced by MLEM2 rule induction algorithm. The input data set of the MLEM2 is a lower or upper approximation of a concept [16]. The LEM2 computes a local covering and then converts it into a rule set. We will quote a few definitions to describe the MLEM2 algorithm [17-20].

A data set is a collection of cases, each case is characterized by attributes and a decision $d$. The value of an attribute $a$ for case $x$ will be denoted by $a(x)$. A block of an attribute-value pair $t=(a, v)$, where $\mathrm{v}$ is the value $a(x)$ of an attribute $a$ for some case $x$, will be denoted by $[t]$.

Let $X$ be a nonempty lower or upper approximation of a concept $[(d, w)]$, where $w$ is the value of $d$ for some case $x$. Set $X$ depends on a set $T$ of attributevalue pairs $t=(a, v)$ if and only if

$$
\emptyset \neq[T]=\bigcap_{t \in T}[t] \subseteq X
$$

Set $T$ is a minimal complex of $X$ if and only if $X$ depends on $T$ and no proper subset $T^{\prime}$ of $T$ exists such that $X$ depends on $T^{\prime}$. Let $\mathcal{T}$ be a nonempty collection of nonempty sets of attribute-value pairs. Then $\mathcal{T}$ is a local covering of $X$ if and only if the following conditions are satisfied:

- each member $T$ of $\mathcal{T}$ is a minimal complex of $X$,

$-\bigcup_{t \in \mathcal{T}}[T]=X$, and 
$-\mathcal{T}$ is minimal, i.e., $\mathcal{T}$ has the smallest possible number of members.

MLEM2 processes numerical attributes differently than symbolic attributes. For numerical attributes MLEM2 sorts all values of a numerical attribute. Then it computes cutpoints as averages for any two consecutive values of the sorted list. For each cutpoint $q$ MLEM2 creates two blocks, the first block contains all cases for which values of the numerical attribute are smaller than $q$, the second block contains remaining cases, i.e., all cases for which values of the numerical attribute are larger than $q$. The search space of MLEM2 is the set of all blocks computed this way, together with blocks defined by symbolic attributes. Finally, MLEM2 simplifies rules by merging intervals for numerical attributes.

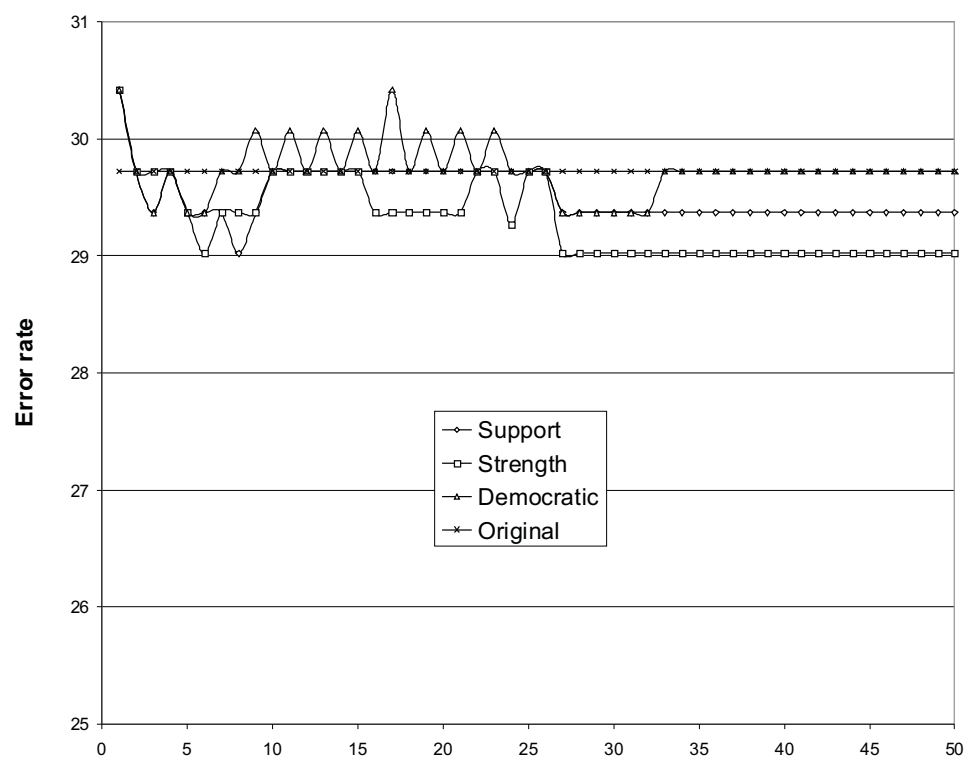

Fig. 1. Breast cancer data set (from Slovenia)

\section{LERS classification system}

This method is based on three parameters: strength, support and matching factor. Note that rule strength and support were introduced in [21]. Matching factor is an original idea of the LERS classification system.

First, the LERS classification system tries to match the case against all rules from the rule set. Each rule is associated with the strength that is equal to the number of training cases that are well classified by this rule. With any concept its support is computed as follows 


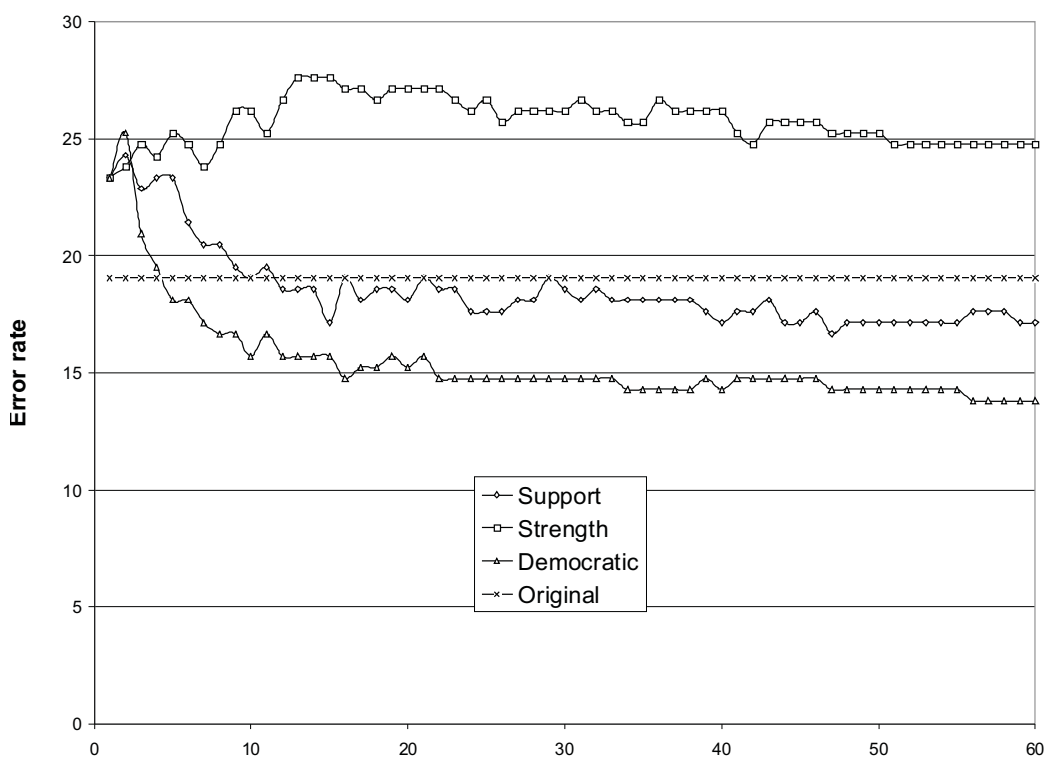

Fig. 2. Image segmentation

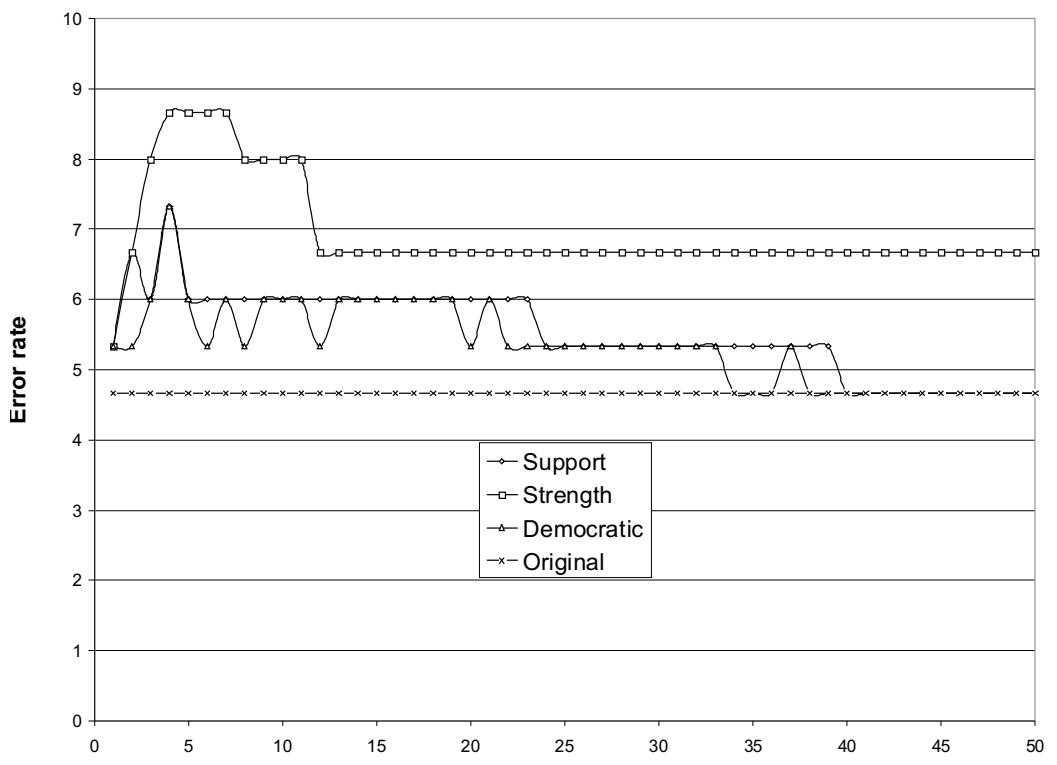

Fig. 3. Iris data set 


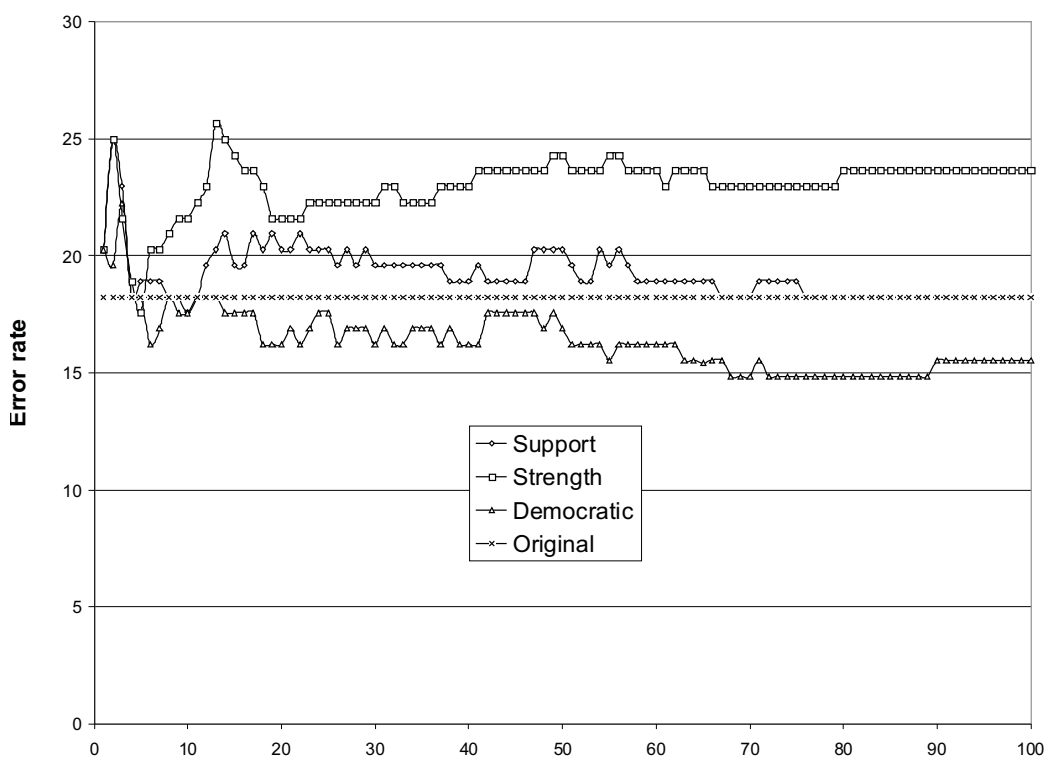

Fig. 4. Lymphography data set

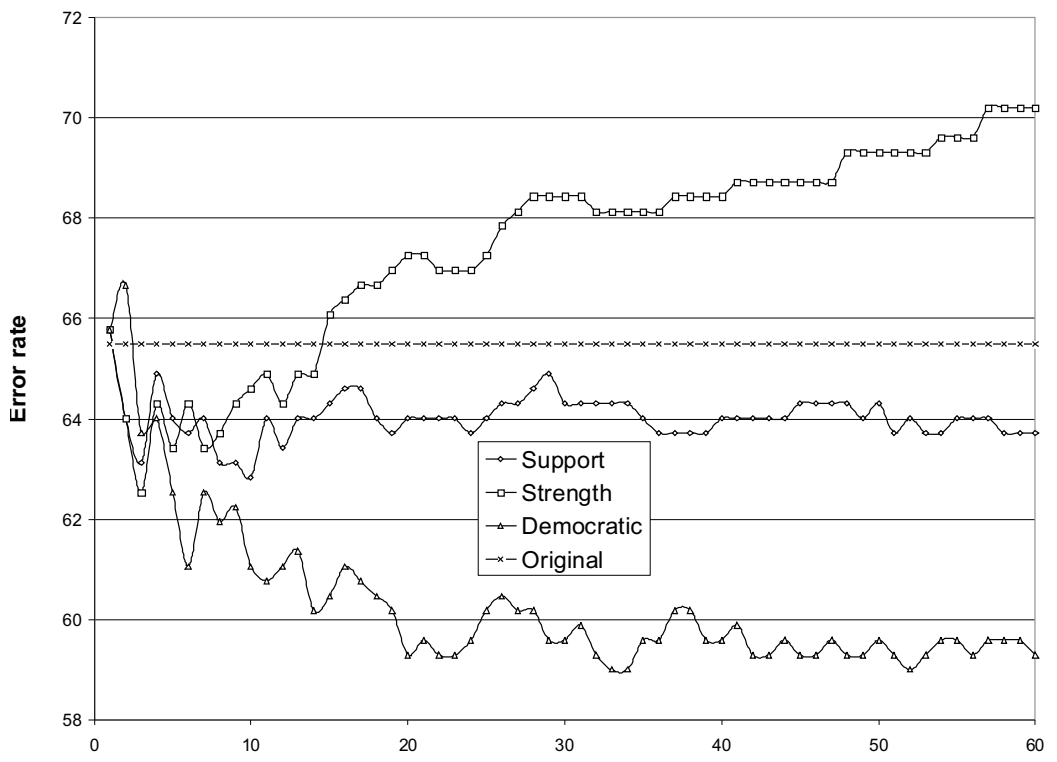

Fig. 5. Primary tumor data set 


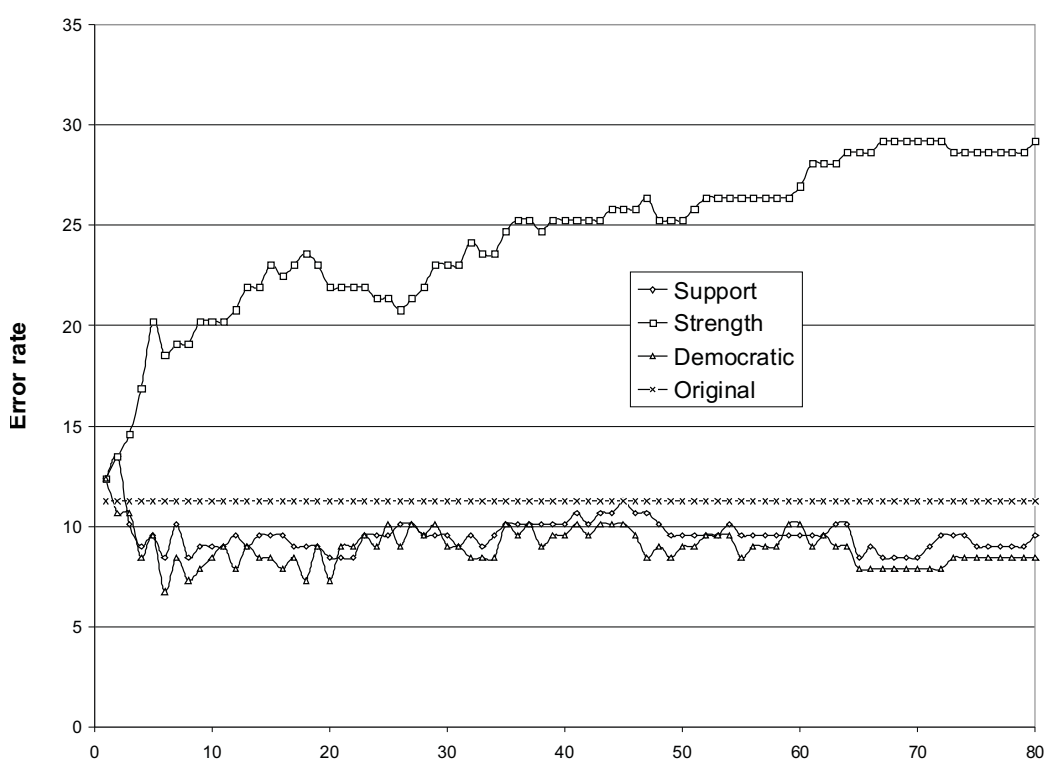

Fig. 6. Wine recognition data set

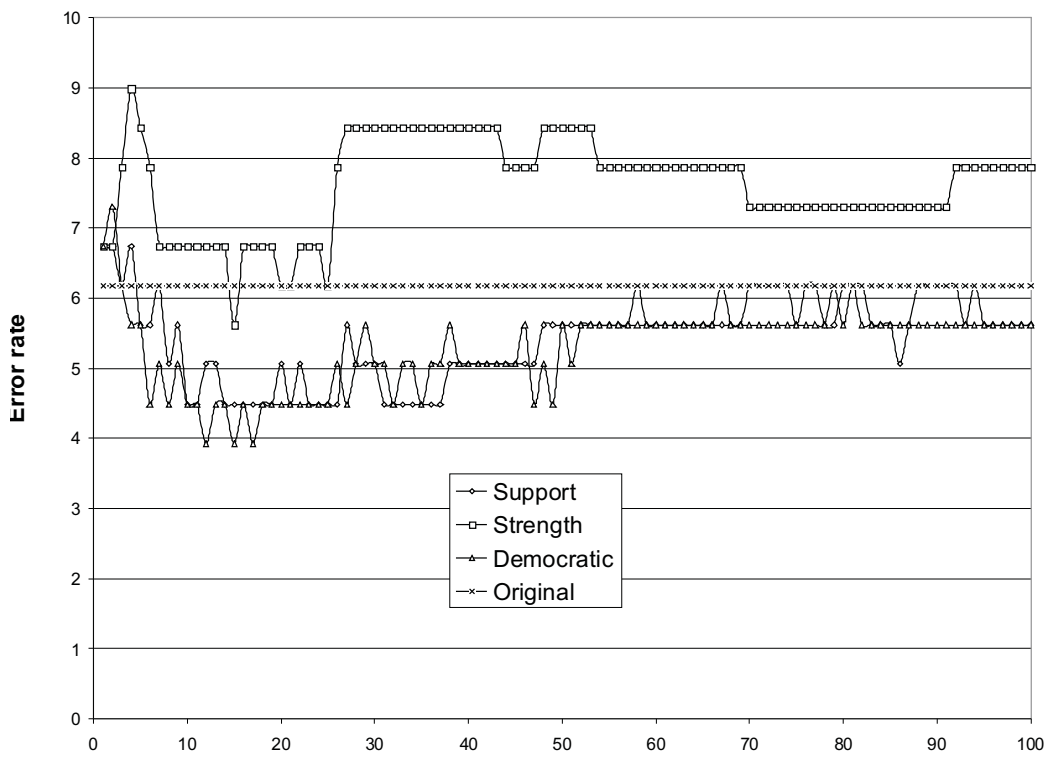

Fig. 7. Wine pre-discretized data set 


$$
\sum_{\text {matching rules } r \text { describing } C} \operatorname{Strength}(r)
$$

The case is classified as a member of the concept with the largest support. If complete matching of a case against the rule set is impossible, all partially matched rules are identified and any concept is associated with a modified support

$$
\sum_{\text {partially matching rules } r \text { describing } C} \text { Matching_factor }(r) * \operatorname{Strength}(r)
$$

where Matching_factor is defined as the ratio of the number of matched attributevalue pairs of $r$ with a case to the total number of attribute-value pairs of $r$. It is possible that a case does not match any rule, even partially. Such a case is not classified. The corresponding rule set abstains from voting. This idea was also explored in [5].

Table 1. Error rates for the ensemble size equal to 100

\begin{tabular}{lrrrr}
\hline \multirow{2}{*}{ Data set } & \multicolumn{2}{c}{ Ensemble voting methods } & \multirow{2}{*}{$\begin{array}{c}\text { Original } \\
\text { data set }\end{array}$} \\
\cline { 2 - 4 } & Support & Strength & Democratic & 4.55 \\
Bankruptcy & 4.55 & 6.06 & 4.55 & 29.72 \\
Breast cancer (Slovenia) & 29.37 & 29.37 & 29.72 & 21.12 \\
Breast cancer (Wisconsin) & 17.92 & 17.92 & 17.92 & 34.78 \\
BUPA & 39.13 & 42.03 & 33.91 & 31.08 \\
Echocardiogram & 27.03 & 31.08 & 29.73 & 30.84 \\
Glass Identification & 34.11 & 45.33 & 28.04 & 17.42 \\
Hepatitis & 16.77 & 20.65 & 14.58 & 35.45 \\
Horse colic & 37.12 & 40.13 & 33.44 & 4.84 \\
House of Representatives & 4.38 & 5.76 & 4.61 & 19.05 \\
Image segmentation & 16.19 & 27.14 & 14.29 & 4.67 \\
Iris & 4.67 & 6.67 & 4.67 & 18.24 \\
Lymphography & 18.24 & 23.65 & 15.54 & 31.77 \\
Pima & 33.33 & 34.38 & 30.47 & 65.49 \\
Primary tumor & 64.31 & 70.80 & 60.18 & 37.78 \\
Postoperative patient & 28.89 & 28.89 & 33.33 & 11.24 \\
Wine recognition & 9.55 & 28.09 & 7.30 & \\
\hline
\end{tabular}




\section{Ensemble voting methods}

We used ten-fold cross validation, so for every data set we computed ten pairs of large $(90 \%)$ training data sets and ten small (10\%) testing data sets. For every training data set 100 bootstrap samples were computed and for every such a sample the corresponding rule set was induced by MLEM2. For every testing case, for all applicable rule sets, the LERS classification method was applied.

Thus, all testing cases were associated with dominant supports, or dominant modified supports, or were labeled as not classified (if even partial matching was impossible). Then we used three different voting methods for ensemble voting.

The first method was based on the support, i.e., each rule set voted with the support (modified support) for the case, for any concept all supports were added, and the concept with the largest sum of all corresponding supports was the winner. In the second method, the choice of the concept was determined by the largest support among rule sets. In the third method, democratic voting, supports of rule sets were converted either into number one if the rule set support was greater than zero, or the corresponding rule set was not voting at all (if the corresponding case was not classified by the rule set).

\section{$5 \quad$ Experiments}

Our experiments were conducted on 16 data sets. All of these data sets, with the exception of Bankruptcy, are available on the University of California at Irvine Machine Learning Repository. The Bankruptcy data set is a well-known data set used by E. Altman to predict a bankruptcy of companies. Some of these data sets: BUPA, Glass identification, Hepatitis, Image segmentation and Pima were pre-discretized using the discretization method based on agglomerative cluster analysis [22].

Results of our experiments, for the size of the ensemble equal to 100, are presented in Table 1 . All of these error rates are results of ten-fold cross-validation. The column Original data set in Table 1 represents the error rates, computed using 10-fold cross-validation, for the original data sets.

Another important feature of bagging is stability. When the size of the ensemble increases, starting from some number, the error rate becomes approximately constant, see Figures 1-7. It is clear that ensemble voting based on strength is not as stable as remaining two voting methods, again, see Figures $1-7$. Note that for the Breast cancer data set (Figure 1) it seems that voting based on strength is stable for the ensemble of size 27 and greater (and that the error rate is $29.02 \%$, the smallest value among our three voting methods). It is misleading since for the ensemble of size 100 the error rate is the same as for the support method, i.e., 29.37\%. Figures 6 and 7 present the same Wine data set, but in Figure 6 this data set had numerical attributes while in Figure 7 this data set was pre-discretized using an agglomerative cluster analysis method of discretization [22], i.e., was symbolic. For the symbolic data set all three ensemble voting methods were more stable. 
During our experiments all ensembles were growing incrementally, with adding a new rule set to the existing rule sets. All three methods of ensemble voting were conducted on the same ensembles (the other possibility would be to create a sequence of ensembles separately for every voting method).

\section{Conclusions}

Results of our experiments show that ordinary, democratic voting is - in the most cases - the best approach to ensemble voting. However, the Wilcoxon matchedpairs signed rank test shows that the democratic voting is not significantly better than voting based on support (two-tailed test, $5 \%$ significance level). On the other hand, voting based on strength is the worst approach to ensemble voting among our three explored methods.

For some data sets, such as Bankruptcy and Iris, bagging did not improve error rate. Most likely, it happens for high quality data sets.

In our experiments all rule sets were certain, i.e., they were induced from concept lower approximations. We are planning to compare certain rules with possible rules combined with bagging, in the future.

\section{References}

1. Breiman, L.: Bagging predictors. Machine Learning 24 (1996) 123-140

2. Freund, Y., Schapire, R.E.: Experiments with a new boosting algorithm. In: Proceedings of the 13-th International Conference on Machine Learning. (1996) $148-156$

3. Breiman, L.: Random forests. Machine Learning 45 (2001) 5-32

4. Bauer, E., Kohavi, R.: An empirical comparison of voting classification algorithms: bagging, boosting, and variants. Machine Learning 36 (1999) 105-139

5. Blaszczynski, J., Stefanowski, J., Zajac, M.: Ensembles of abstaining classifiers based on rule sets. In: Proceedings of the International Symposium on Foundations of Intelligent Systems. (2009) 382-391

6. Roiger, R.J., Geatz, M.W.: Data Mining. A Tutorial-Based Primer. AddisonWesley, Boston, MA (2003)

7. Stefanowski, J.: The bagging and $n^{2}$-classifiers based on rules induced by MODLEM. In: Proceedings of the Fourth International Conference on Rough Sets and Current Trends in Computing. (2004) 488-497

8. Stefanowski, J.: On combined classifiers, rule induction and rough sets. Transactions on Rough Sets 6 (2007) 329-350

9. Weiss, S.M., Indurkhya, N.: Predictive Data Mining. A Practical Guide. Morgan Kaufmann Publ., San Francisco, CA (1998)

10. Zenko, B., L., T., Dzeroski, S.: On comparison of stacking with MDTs to bagging, boosting, and other stacking methods. In: Proceedings of the ECML/PKDD 01 Workshop on Integrating Aspects of Data Mining, Decision Support and MetaLearning. (2001) 163-175

11. Wolpert, D.: Stacked generalization. Neural Networks 5 (1992) 241-260

12. Gama, J.: Combining classifiers by constructive induction. In: Proceedings of the 10-th European Conference on Machine Learning. (1998) 178-189 
13. Quinlan, J.R.: C4.5: Programs for Machine Learning. Morgan Kaufmann Publishers, San Mateo, CA (1993)

14. Hall, L.O., Bowyer, K.W., Banfield, R.E., Bhadoria, D., Kegelmeyer, W.P., Eschrich, S.: Comparing pure prallel ensemble creation techniques against bagging. In: Proceedings of the IEEE International Conference on Data Mining. (2003) $533-536$

15. Dietterich, T.G.: An experimental comparison of three methods for constructing ensembles of decision trees: bagging, boosting, and randomization. Machine Learning 40 (2000) 139-157

16. Pawlak, Z.: Rough Sets. Theoretical Aspects of Reasoning about Data. Kluwer Academic Publishers, Dordrecht, Boston, London (1991)

17. Grzymala-Busse, J.W.: LERS - a system for learning from examples based on rough sets. In Slowinski, R., ed.: Intelligent Decision Support. Handbook of Applications and Advances of the Rough Set Theory. Kluwer Academic Publishers, Dordrecht, Boston, London (1992) 3-18

18. Chan, C.C., Grzymala-Busse, J.W.: On the attribute redundancy and the learning programs ID3, PRISM, and LEM2. Technical report, Department of Computer Science, University of Kansas (1991)

19. Grzymala-Busse, J.W.: A new version of the rule induction system LERS. Fundamenta Informaticae 31 (1997) 27-39

20. Grzymala-Busse, J.W.: MLEM2: A new algorithm for rule induction from imperfect data. In: Proceedings of the 9th International Conference on Information Processing and Management of Uncertainty in Knowledge-Based Systems. (2002) 243-250

21. Holland, J.H., Holyoak, K.J., Nisbett, R.E.: Induction. Processes of Inference, Learning, and Discovery. MIT Press, Boston (1986)

22. Chmielewski, M.R., Grzymala-Busse, J.W.: Global discretization of continuous attributes as preprocessing for machine learning. International Journal of Approximate Reasoning 15(4) (1996) 319-331 